\title{
Are Hematologic Patients Trojan Horse for COVID-19?
}

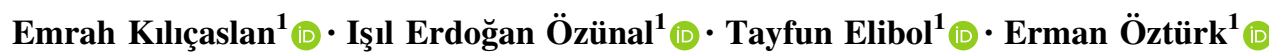

Received: 7 June 2021/Accepted: 5 October 2021/Published online: 15 October 2021

(C) Indian Society of Hematology and Blood Transfusion 2021

\begin{abstract}
Coronavirus disease 2019 (COVID-19) which is caused by severe acute respiratory distress syndrome virus (SARS-CoV-2) continues to affect people all around the world. This study aimed to compare the SARS-CoV-2 viral shedding time between patients diagnosed with hematologic diseases (HD) and a control group. A total of 110 patients were enrolled in this retrospective study; 55 patients with a diagnosis of HD and 55 sex and comorbidity matched controls without a diagnosis of HD, who caught COVID-19 at the same period. Thirty-eight patients were hospitalized in each group. Viral shedding time, COVID-19 severity, need for intensive care unit support, and mortality rates were compared between groups. Median viral shedding time was 24 days in hospitalized HD patients and 12 days in the hospitalized control group $(p<0.01)$ as 20 days in outpatient HD patients and 10 days in the outpatient control group $(p=0.02)$. Viral shedding time was longer in severe + critical COVID-19 cases in the whole cohort (median 22 days in severe + critical, and 12 days in mild + moderate) $(p<0.01)$. Severe + critical COVID-19 was more common in the HD group than the control group $(47.2 \%$ versus $25.4 \%$, respectively) $(p=0.017)$. Twenty-five patients were dead in the whole cohort. One patient was in the control group and 24 patients were in the HD group, therefore the mortality rate for the HD group was $43.6 \%$. Because of statistically significant longer viral shedding
\end{abstract}

Iş11 Erdoğan Özünal

isilnoktaerdogan@hotmail.com

1 Division of Hematology, Department of Internal Medicine, Faculty of Medicine, Istanbul Medeniyet University, Istanbul, Turkey time, longer-term isolation may be necessary for hematologic patients diagnosed with COVID-19.

Keywords COVID-19 · SARS-CoV-2 · Hematological diseases $\cdot$ Viral shedding $\cdot$ Treatment

\section{Introduction}

The World Health Organization (WHO) has declared a global pandemic as there are a growing number of cases of coronavirus disease 2019 (COVID-19), resulting in alarmingly high mortality and morbidity in many countries around the world. Although many drugs have been used for the treatment to date, no evidence-based specific treatment has been found yet.

The first COVID-19 case in our country was detected on 11th March 2020. As of February 22, 2021, a total of 2,646,526 cases were detected, and 28,138 patients died due to COVID-19 in the general population. There are different data on the mortality of the disease in the general population in the world. The COVID-19 mortality rate in our country is approximately $1.06 \%$ [1].

COVID-19 may have an attributable course in patients diagnosed with hematologic diseases (HD). Mortality rates may be higher than the normal population in these patient groups $[2,3]$. This can be because of active hematologic malignancy, disease or treatment-induced immunosuppression, or elongation of COVID-19 infection duration associated with prolonged clearance of the virus. Although the time of viral shedding is defined in immunocompetent patients with COVID-19, little is known about this issue in immunocompromised patients [4]. This study aimed to evaluate the COVID-19 viral shedding time and severity in 
hospitalized and outpatient patients diagnosed with HDs and compare this with a control group.

\section{Materials and Methods}

\section{Study Design and Patients}

A total sample size of 102 patients was needed to ensure a one-sided test with a level of significance $\alpha=0.05$ with $80 \%$ power. So, 110 patients were enrolled in this retrospective controlled descriptive single-center study. Fiftyfive patients who were diagnosed with HD, and during routine follow-up contracted the severe acute respiratory distress syndrome virus (SARS-CoV-2) and were diagnosed with COVID-19 between March 2020-February 2021 were enrolled. As a control group, fifty-five sex and comorbidity matched patients diagnosed with COVID-19 between March 2020-February 2021, whose COVID-19 polymerase chain reaction (PCR) test positivity and negativity were documented and did not have a history of hematologic or oncologic disease were randomly enrolled in the study. Data were collected from patient files. The study was approved by the local ethics committee of Istanbul Medeniyet University and approved by the provincial health directorate, Istanbul. The primary endpoint was viral shedding time defined as in days of PCR test positivity duration. Secondary endpoints were COVID19 severity, need for intensive care unit support and/or mechanical ventilation, mortality, and HD or treatment status.

\section{Definitions}

Patients who were newly diagnosed or had relapsed HD were defined as having active HD while patients who had stable disease or who did not have treatment indications (like early-stage chronic lymphocytic leukemia or indolent lymphomas) were defined as having controlled HD. Also, patients whose treatment was recently completed and regarded as clinically responsive but not yet evaluated for remission and patients who achieved complete remission were defined as having controlled HD. Patients who were receiving a combined chemotherapy regimen, hypomethylating agents, monoclonal antibodies, ibrutinib, lenalidomide, or steroid treatment because of HD were defined as having active treatment. According to symptoms of the disease, the severity of COVID-19 was graded as; mild, moderate, severe, and critical as described before [5]. Positive PCR test time was described as a positive test result of COVID-19 PCR in nasopharyngeal (NP) and oropharyngeal (OP) swabs. Patients were monitored to test for PCR during hospitalization or outpatient follow-up. Negative PCR test time was described as achieving two consecutive negative PCR test results. The duration of PCR positivity was calculated in days by subtracting the positive test day from the first negative test day.

Treatment strategies for COVID-19 were similar in the whole cohort as recommended by the ministry of health's COVID-19 recent treatment guidelines in Turkey [6]. This strategy was composed of using an antiviral treatment for symptomatic outpatient patients as well as the addition of low-molecular-weight heparins and steroids to the treatment for hospitalized patients.

COVID-19 viral shedding time, severity, hospitalization, the requirement for intensive care unit support and/or mechanical ventilation, $\mathrm{HD}$, and treatment status were evaluated. Mortality rates, cause of mortality, state of HD at the time of death were evaluated and compared in hospitalized and outpatient patients.

\section{Statistical Analysis}

SPSS (version 22) program was used for all statistical analyses and figure creation. Pearson Chi-square analysis or Fisher exact test was applied for the comparison of categorical variables whereas Mann-Whitney's U test was used for the comparison of continuous variables. Estimates of median PCR test positivity duration were analyzed through Kaplan-Meier analysis using Log Rank test. Type I error was less than or equal to 0.05 .

\section{Results}

A total of 110 patients were enrolled in the study; 55 patients were in the HD group and 55 in the control group. Thirty-eight patients were hospitalized and 17 patients were outpatient cases in both groups. Twenty-nine patients were male $(52.7 \%)$ in the HD group and the median age was 65 years (range: 20-84 years). Twenty-five patients were male $(45.4 \%)$ in the control group and the median age was 54 years (range: $21-92$ years). According to age, there was no statistically significant difference between hospitalized patient groups (median 67.5 years in hospitalized HD group versus 60.5 years in the hospitalized control group, $p=0.204)$. The outpatient control group was younger than the outpatient HD group $(p<0.01)$. The patients' characteristics were summarized in Table 1. Fiftysix patients had comorbidities like hypertension, diabetes, atherosclerotic coronary artery disease, atrial fibrillation, or chronic obstructive pulmonary disease in the whole cohort. The frequency of occurrence of these comorbidities was similar in both HD and control groups. In the HD group, 50 of 55 patients $(91 \%)$ had a malignant hematologic disease 
Table 1 Characteristics of patients

\begin{tabular}{|c|c|c|}
\hline & $\begin{array}{l}\text { Patients with hematologic diseases } \\
(\mathrm{N}=55)\end{array}$ & $\begin{array}{l}\text { Control group } \\
(\mathrm{N}=55)\end{array}$ \\
\hline Male & $29(52.7 \%)$ & $25(45.4 \%)$ \\
\hline Hospitalized & $38(69 \%)$ & $38(69 \%)$ \\
\hline Age (median/range) years & $65(20-84)$ & $54(21-92)$ \\
\hline Hospitalized & 67.5 & 60.5 \\
\hline Outpatient & 55 & 30 \\
\hline $\begin{array}{l}\text { Comorbidity (number of patients having one or more of either; HT, DM, CAD, } \\
\text { COPD, AF) }\end{array}$ & $29(52.7 \%)$ & $27(49 \%)$ \\
\hline \multirow[t]{7}{*}{ Hematologic diagnosis } & 18 NHL $(32.7 \%)$ & - \\
\hline & 6 ALL $(11 \%)$ & \\
\hline & $6 \mathrm{MM}(11 \%)$ & \\
\hline & 6 CLL $(11 \%)$ & \\
\hline & 5 AML $(9 \%)$ & \\
\hline & $4 \operatorname{MDS}(7 \%)$ & \\
\hline & 10 others & \\
\hline Active disease & $35(63.6 \%)$ & - \\
\hline Receiving treatment & $30(54.5 \%)$ & - \\
\hline \multicolumn{3}{|l|}{ COVID-19 severity } \\
\hline Mild + moderate & $29(52.7 \%)$ & $41(74.5 \%)$ \\
\hline Severe + Critical & $26(47.2 \%)$ & $14(25.4 \%)$ \\
\hline Intensive care unit support & $21(38.1 \%)$ & $6(10.9 \%)$ \\
\hline Mechanical ventilation & $18(32.7 \%)$ & $3(5.4 \%)$ \\
\hline
\end{tabular}

HT, hypertension; DM, diabetes mellitus; CAD, coronary artery disease; COPD, chronic obstructive pulmonary disease; AF, atrial fibrillation; NHL, non-Hodgkin lymphoma; ALL, acute lymphoblastic leukemia; MM, multiple myeloma; CLL, chronic lymphocytic leukemia; AML, acute myeloid leukemia; MDS, myelodysplastic syndrome

and the most common diagnosis was non-Hodgkin lymphoma (NHL) with a rate of $32.7 \%$. Five patients had nonmalignant diseases as pure red cell aplasia, thrombotic thrombocytopenic purpura, immune thrombocytopenia, and aplastic anemia.

\section{Viral Shedding Time}

Median viral shedding time was 24 days (range between 4 and 92 days) in hospitalized HD patients and 12 days (range between 5 and 31 days) in the hospitalized control group ( $p<0.01)$ (Table 2). Median viral shedding time was 20 days (range between 9 and 62 days) in outpatient HD patients and 10 days (range between 6 and 20 days) in

Table 2 Viral shedding time according to groups

\begin{tabular}{llll}
\hline & $\begin{array}{l}\text { Patients with } \\
\text { hematologic diseases } \\
(\mathrm{N}=55) \text { (days, median/ } \\
\text { range })\end{array}$ & $\begin{array}{l}\text { Control group } \\
(\mathrm{N}=55)(\text { days } \\
\text { median/range) }\end{array}$ & $p$ \\
\hline Hospitalized & $24(4-92)$ & $12(5-31)$ & $<0.01$ \\
Outpatient & $20(9-62)$ & $10(6-20)$ & 0.02 \\
\hline
\end{tabular}

the outpatient control group ( $p=0.02$ ) (Fig. 1). There was not a statistically significant difference in median viral shedding time between hospitalized and outpatient hematologic patients (24 vs 20 days respectively, $p=0.261$ ).

\section{COVID-19 Severity}

COVID-19 severity was compared between groups by classifying patients according to the COVID-19 course as mild + moderate and severe + critical. In the whole cohort, 70 patients $(63.7 \%)$ had mild + moderate COVID19 while 40 patients had severe + critical COVID-19. Mild + moderate disease was seen in 41 patients $(74.5 \%)$ in control group and 29 patients $(52.7 \%)$ in HD group. Therefore mild + moderate COVID-19 was more common in the control group than in the HD group $(p=0.017)$ (Table 1).

Severe + critical COVID-19 was more common in the HD group (26 versus 14 patients). According to these findings, the COVID-19 course was more severe in the HD group ( $p=0.017$ ) (Table 1$)$. In the whole cohort, median viral shedding time was 22 days versus 12 days in 
Fig. 1 Viral shedding time between groups according to hospitalization
Fig. 2 Viral shedding time according to COVID-19 severity in the whole cohort
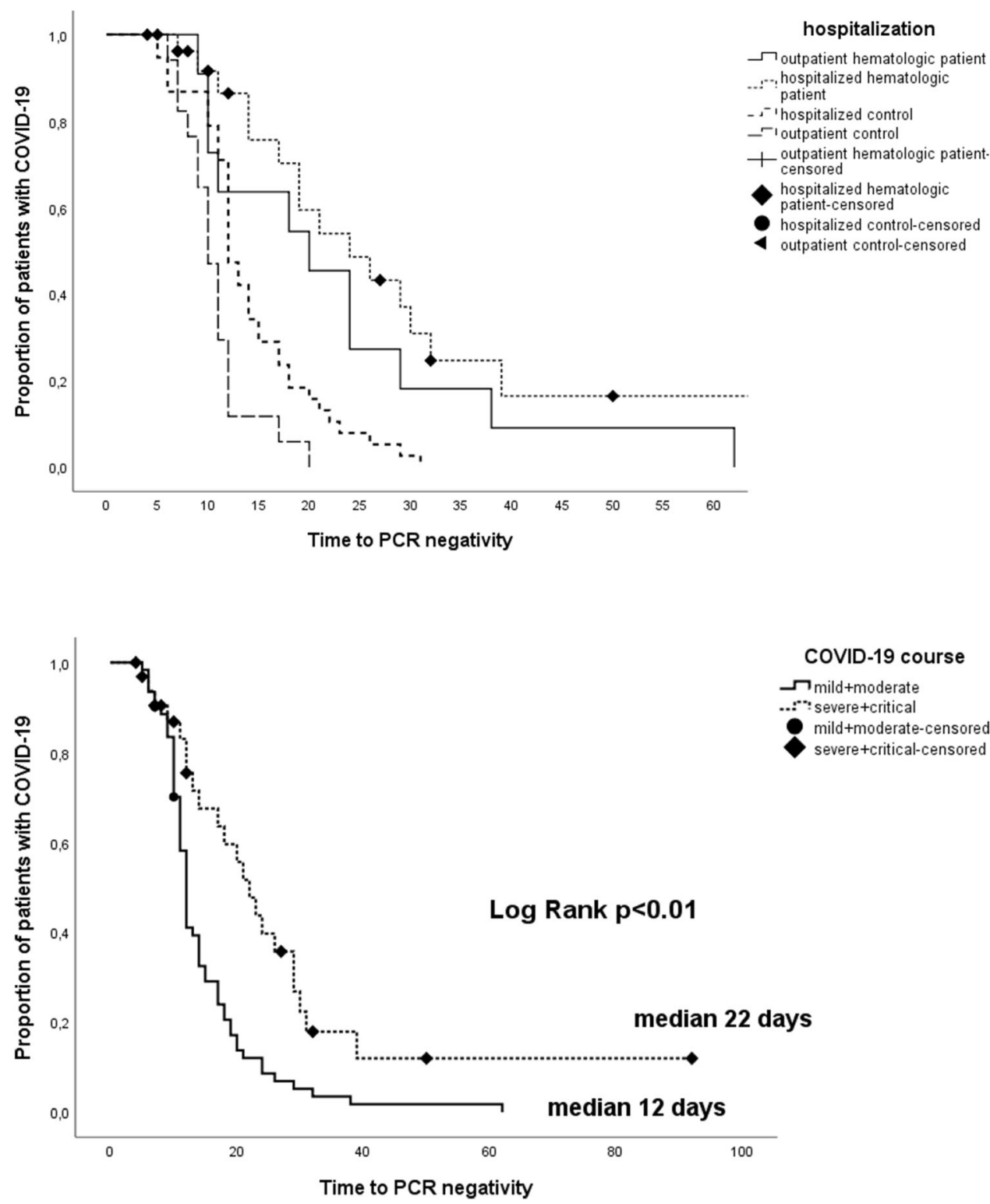

COVID-19 course

$\neg$ mild+moderate severe+critical

mild+moderate-censored

severe+critical-censored severe + critical and mild + moderate COVID-19 respectively ( $p<0.01$ ) (Fig. 2).

\section{Intensive Care Unit Support and/or Mechanical Ventilation}

Intensive care unit support and mechanical ventilation were more common in the HD group. In the whole cohort, 27 patients $(24.5 \%)$ required intensive care unit support, 21 of them $(77.7 \%)$ were in the HD group $(p=0.001)$ and 21 patients $(19 \%)$ required mechanical ventilation 18 of whom $(85.7 \%)$ were in the HD group $(p<0.01)$.

\section{Disease Status}

In the HD group, 35 patients $(63.6 \%)$ had an active hematologic disease while 20 patients $(36.4 \%)$ had controlled disease. In the whole cohort, viral shedding time was the longest in hospitalized HD patients who had the active disease (Fig. 3). Patients with active HD had a median viral shedding time of 24 days whereas patients without active HD had 20 days $(p=0.193)$.

\section{Mortality}

Twenty-five patients $(22.7 \%)$ were dead in the whole cohort (13 male, 12 female patients, $p=0.741$ ). One patient was in the control group and 24 patients were in the HD group, therefore the mortality rate for the HD group 
Fig. 3 Viral shedding time according to hematologic disease status

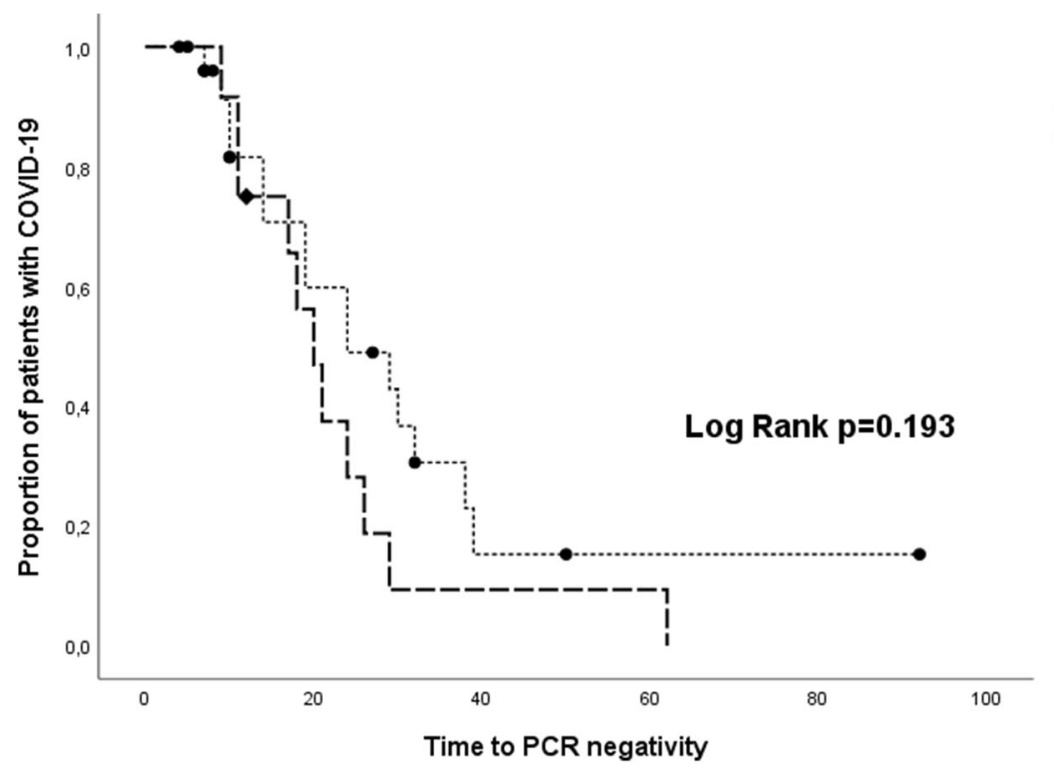

was $43.6 \%$. Twenty of these patients in the HD group had active HD at the time of death. Ten male patients and 11 female patients were died because of COVID-19 in the HD group $(p=0.537)$. Three patients in the HD group were died because of HD.

\section{Discussion}

Detection of SARS-CoV-2 viral RNA in NP or OP swab is important for the diagnosis and determining the contagiousness of COVID-19, but little is known about the length of stay of the virus in immunocompromised hematologic patients. In this study, we examined factors that may have had an impact on viral shedding time of SARSCoV-2 in hematologic patients and compare these with a control group.

Median viral shedding time was significantly longer in hematologic patients than the control group in both hospitalized and outpatient patients ( 24 vs. 12 days in hospitalized patients). There was no statistically significant difference in median viral shedding time between hospitalized and outpatient hematologic patients ( 24 vs 20 days respectively). Median viral shedding time was the longest in patients who had active HD, but for this data, there was no statistically significant difference between patients with active and controlled HD (24 days vs. 20 days, respectively).

There are publications in the literature revealing different data about the duration of SARS-CoV-2 viral RNA shedding in NP and/or OP swabs [7]. Fang and Zhang reported the median duration of positive NP PCRs in patients who were admitted to the intensive care unit as 22.25 ( \pm 3.62) days, compared to $15.67( \pm 6.68)$ days in patients without the need for intensive care unit support [8]. Feng and Li stated that; male sex, receiving lopinavir/ ritonavir treatment within 7 days from illness onset, and receiving systemic glucocorticoid therapy were independent factors associated with prolonged viral shedding time in patients without a diagnosis of hematologic malignancy [9]. Zhu and Gong reported a longer time of viral shedding in patients with renal transplantation compared to controls $(28.4+9.3$ days vs. $12.2+4.6$ days, $p<0.01) \quad$ [10]. Aydillo and Gonzalez-Reiche detected viral RNA in NP samples up to 78 days after the onset of symptoms (interquartile range, 24 to 64 days) in 20 immunocompromised patients [4]. Wang and Van Oekelen reported the median time to PCR negativity as 43 days (range 19-68 days) after the first positive PCR test in 20 patients with plasma-cell disorders [11]. A recent study showed median time to PCR negativity in patients with hematologic malignancies as 17 days (7-49 days) [12]. There are also publications in the literature in the form of case reports about prolonged viral shedding time in hematologic malignancies $[13,14]$.

In our study, the severe + critical COVID-19 course was related to longer viral shedding time in the whole cohort (median 22 days in severe + critical vs 12 days in mild + moderate, $p<0.01$ ) (Fig. 2), and severe + critical COVID-19 course was more common in the HD group $(47.2 \%)$ than the control group $(25.4 \%)(p=0.017)$. In addition, the requirement for intensive care unit support and mechanical ventilation was higher in the HD group. Similar to our results, García-Suárez and De La Cruz reported COVID-19 clinical severity as severe/critical in $62 \%$ of patients diagnosed with hematologic malignancies [15]. HD and the treatment of HD can worsen the immune system responses and can make patients more vulnerable to 
COVID-19 disease [16]. This may be the reason for experiencing COVID-19 more severely in patients diagnosed with HD. Based on these findings, the prolonged viral shedding time can be attributed to the underlying HD and the severity of the COVID-19 course.

Different COVID-19 mortality rates in hematologic patients have been reported in the literature; a mortality rate of $33 \%$ was reported from Spain, and 37\% from Italy $[15,17]$. Other publications specified $40 \%$ and $62 \%$ mortality rates in hospitalized COVID-19 patients with hematologic malignancies [18, 19]. A recent study from Turkey was published by Yigenoglu and Ata, which included 770 patients diagnosed with hematologic malignancies and COVID-19 [3]. The distribution of patients by hematologic diagnosis in this study was as; $30.1 \%$ NHL, $15.7 \%$ myeloproliferative neoplasms (MPN), $19.7 \%$ myelodysplastic syndromes, $10.4 \%$ multiple myeloma, $7.8 \%$ acute leukemias, and $16.2 \%$ others, and mortality due to COVID19 was reported to be $13.8 \%$ [3]. Comparing to Yigenoglu and Ata's s results; NHL rates were similar, acute leukemias were higher and MPNs were lower in our study (Table 1), and the mortality rate of the HD group was $43.6 \%$. The difference in mortality rates between these studies may be due to the heterogeneous distribution of patients' hematologic diagnosis and COVID-19 severity.

Although the literature stated as the male sex increases COVID-19 mortality, we did not come to this conclusion in hematologic patients [20]. Thirteen male and 12 female patients died in the entire cohort. One patient was in the control group. There was no significant difference between sex in terms of death in the HD group ( $p=0.741)$.

Several limitations of our study deserve to be mentioned. We could not be able to perform viral culture in patients with prolonged viral RNA shedding in NP and/or OP swab. So precise data on whether the cause of PCR positivity is due to living viruses or dead virus fragments cannot be given. Also, we could not be able to state the onset and duration of symptoms related to COVID-19 in all patients because some of them-even in the hospitalized HD group-were asymptomatic and PCR positivity was detected just on account of during a routine check-up before starting chemotherapy. Due to the small number of patients, it was not possible to categorize the patients according to the treatment received for HD. Patient groups incorporate a heterogeneous population with various HDs; so further studies in patients with specific HD are needed, the type of disease status may impress the clinical course of COVID-19.

In our study, the median viral shedding time was significantly longer in the HD group compared to the controls, and there was no statistically significant difference between outpatient and hospitalized hematologic patients. According to the Centers for Disease Control and Prevention
(CDC), a quarantine period of 14 days is endorsed for people who exposed to the virus, and an isolation period of 10 days after the onset of symptoms is recommended for most adults, but this may warrant up to 20 days for immunocompromised patients [21]. We found out that even in hospitalized hematologic patients it can be difficult to determine the time of the onset of symptoms because these patients can be asymptomatic. Also, we observed a period up to 7 or 10 days between the positive PCR test result and the onset of symptoms related to COVID-19 disease in several hematologic patients. We also observed that some patients developed re-infection as cited by Fontana LM [7]. This is significant because longer than recently recommended isolation may be required for these patients concerning public health. In this context, hematologic patients require a more specific approach in terms of COVID-19 management compared to the normal population. It remains unclear whether chemotherapy and/or immunosuppressive therapy should be delayed or not in these patient groups. Studies involving more patients are needed to clarify this issue.

Acknowledgements We thank all of our colleagues and also patients for their assistance.

Author Contributions EK, IEÖ, TE, and EÖ performed the research and analyzed and interpreted the data. EK and IEÖ created the figures and wrote the manuscript. TE performed the data analyses and EÖ reviewed the manuscript. All authors reviewed the results and approved the final version of the manuscript.

Funding This paper was not funded.

\section{Declarations}

Conflicts of interest No potential conflict of interest was reported by the author(s).

Data Availability The datasets used and/or analyzed during the current study are available from the corresponding author on reasonable request.

Ethical Approval and Consent to Participate The study was approved by the local ethics committee of Medeniyet University and approved by the provincial health directorate, Istanbul, and per the ethical standards formulated in the Declaration of Helsinki. Informed consent was obtained from all patients.

\section{References}

1. Ministry of Health T. COVID-19 Information Platform 2021. Available from: https://covid19.saglik.gov.tr/TR-66935/genelkoronavirus-tablosu.html

2. Lee LY, Cazier J-B, Starkey T et al (2020) COVID-19 prevalence and mortality in patients with cancer and the effect of primary tumour subtype and patient demographics: a prospective cohort study. Lancet Oncol 21(10):1309-1316 
3. Yigenoglu TN, Ata N, Altuntas F et al (2021) The outcome of COVID-19 in patients with hematological malignancy. J Med Virol 93(2):1099-1104. https://doi.org/10.1002/jmv.26404

4. Aydillo T, Gonzalez-Reiche AS, Aslam S et al (2020) Shedding of viable SARS-CoV-2 after immunosuppressive therapy for cancer. N Engl J Med 383(26):2586-2588

5. He HW, Chen L et al (2020) COVID-19 in persons with haematological cancers. Leukemia 34(6):1637-1645

6. Ministry of Health T. COVID-19 Adult Patient Treatment 2020. Available from: https://covid19.saglik.gov.tr/TR-66926/eriskinhasta-tedavisi.html

7. Fontana L, Villamagna AH, Sikka MK et al (2020) Understanding viral shedding of SARS-CoV-2: review of current literature. Infect Control Hosp Epidemiol, 1-35

8. Fang Z, Zhang Y, Hang C et al (2020) Comparisons of viral shedding time of SARS-CoV-2 of different samples in ICU and non-ICU patients. J Infect 81(1):147-178

9. Feng Z, Li J, Yao S et al (2020) Clinical factors associated with progression and prolonged viral shedding in COVID-19 patients: a multicenter study. Aging Dis 11(5):1069

10. Zhu L, Gong N, Liu B et al (2020) Coronavirus disease 2019 pneumonia in immunosuppressed renal transplant recipients: a summary of 10 confirmed cases in Wuhan. China Eur Urol 77(6):748-754

11. Wang B, Van Oekelen O, Mouhieddine TH et al (2020) A tertiary center experience of multiple myeloma patients with COVID-19: lessons learned and the path forward. J Hematol Oncol 13(1):1-12

12. Borah P, Mirgh S, Sharma SK et al (2021) Effect of age, comorbidity and remission status on outcome of COVID-19 in patients with hematological malignancies. Blood Cells, Mole 87:102525

13. Avanzato VA, Matson MJ, Seifert SN et al (2020) Case Study: prolonged infectious SARS-CoV-2 shedding from an asymptomatic immunocompromised cancer patient. Cell Biosci 183:1901
14. Karataş A, İnkaya AÇ, Demiroğlu H et al (2020) Prolonged viral shedding in a lymphoma patient with COVID-19 infection receiving convalescent plasma. Transfus Apher Sci 59(5):102871

15. García-Suárez J, De La Cruz J, Cedillo Á et al (2020) Impact of hematologic malignancy and type of cancer therapy on COVID19 severity and mortality: lessons from a large population-based registry study. J Hematol Oncol 13(1):1-12

16. Kuderer NM, Choueiri TK, Shah DP et al (2020) Clinical impact of COVID-19 on patients with cancer (CCC19): a cohort study. Lancet (London, England) 395(10241):1907-1918. https://doi. org/10.1016/s0140-6736(20)31187-9.PubMedPMID: 32473681;PubMedCentralPMCID:PMCPMC7255743.eng

17. Passamonti F, Cattaneo C, Arcaini L et al (2020) Clinical characteristics and risk factors associated with COVID-19 severity in patients with haematological malignancies in Italy: a retrospective, multicentre, cohort study. Lancet Haematol 7(10):e737e745

18. Piñana JL, Martino R, García-García I et al (2020) Risk factors and outcome of COVID-19 in patients with hematological malignancies. Exp Hematol Oncol 9(1):21. https://doi.org/10. 1186/s40164-020-00177-z

19. Li Q, Cao Y, Chen L et al (2020) Hematological features of persons with COVID-19. Leuk Lymphoma 34(8):2163-2172

20. Albitar O, Ballouze R, Ooi JP et al (2020) Risk factors for mortality among COVID-19 patients. Diabet Res Clin Pract 166:108293

21. Interim Guidance on Ending Isolation and Precautions for Adults with COVID-19. Available from: https://www.cdc.gov/cor onavirus/2019-ncov/hcp/duration-isolation.html

Publisher's Note Springer Nature remains neutral with regard to jurisdictional claims in published maps and institutional affiliations. 